\title{
Investigating gaps on Agile Improvement Solutions and their successful adoption in industry projects - A systematic literature review
}

\author{
Arthur Freire*, André Meireles ${ }^{\dagger}$, Gleyser Guimarães ${ }^{\ddagger}$, Mirko Perkusich ${ }^{\S}$, \\ Raissa da Silva*, Kyller Gorgônio*, Angelo Perkusich* and Hyggo Almeida* \\ * Intelligent Software Engineering (ISE) Group, Federal University of Campina Grande \\ Emails: \{arthurfreire, raissasilva\}@ copin.ufcg.edu.br, \\ \{kyller, hyggo\}@dsc.ufcg.edu.br, and perkusic@dee.ufcg.edu.br \\ $\dagger$ Federal University of Ceará - Email: andre@crateus.ufc.br \\ $\ddagger$ Federal University of Campina Grande — Email: gleyser@ copin.ufcg.edu.br \\ $\S$ Federal Institute of Paraiba — Email: mirko.perkusich@ifpb.edu.br
}

\begin{abstract}
-
Background: The focus of Agile software development (ASD) is different than plan-driven development, requiring new software process improvement (SPI) paradigms.

Objective: To identify and synthesize the possible gaps of Agile improvement solutions (AIS) given their focus on people factors, report of successful adoption in industry projects and availability of tool support.

Method: We applied a Systematic Literature Review of studies published up to (and including) 2017 through backward and forward snowballing given a start set.

Results: In total, we evaluated 55 papers, of which 44 included AIS and the main findings are: 1) 26 consider teamwork factors; 2) 21 were applied on industry; 3) 10 out of these 21 presented evidence of increase in company performance; and 4) 19 of the solutions are for the purpose of adoption, 18 for assessment and 8 are maturity models.

Conclusion: The main implication for this research is a need for more and better empirical studies documenting and evaluating AIS. For the industry, the review provides a map of current AIS approaches and can be used as a starting point to adopt agile SPI.
\end{abstract}

Index Terms-Agile, Systematic Literature Review, Maturity Model, Assessment, Adoption, Tailoring

\section{INTRODUCTION}

Agile software development (ASD) methods have gained much attention throughout the last years due to the need of adaptability and flexibility in software projects [1]. ASD is considered an alternative approach to plan-driven development because it promises some benefits if compared to these approaches such as delivery of business value in short iterations. Moreover, it focuses more on people and their interactions instead of processes and tools.

According to Salo \& Abrahamsson [2], since the focus of agile methods is different than plan-driven development processes, there is a need of new software process improvement (SPI) (i.e., initiatives that can be used in software organizations to mature their operations [3]).
One possibility to use ASD methods is by tailoring a particular method to fit a given context. In some contexts, ASD is implemented along with SPI initiatives based on the ISO/IEC 33001 international standard or the Capability Maturity Model Integration for Development (CMMI-DEV) [4], [5], [6], [7], [8], [9], [10]. However, these models require that processes be formally defined and controlled, which is not usual practice in ASD [11].

Fontana et al. [11] hypothesize that, given the current definition of maturity defined by CMMI-DEV, agile teams could never achieve maturity without shifting their focus from process to people. Given this, they proposed a definition for ASD maturity that includes not only the definition and improvement of processes, but also people factors such as collaboration, communication, commitment, care, sharing and self-organization.

We defined a terminology for some terms that we use throughout the paper to ease the reading:

- Maturity: related to maturity itself, agility or improvement;

- Maturity Model: according to Kohlegger et al. [12], maturity models are instruments used to rate capabilities, and based on this rating, initiatives can be implemented to improve the maturity of an element - a person, an object or a social system. In this paper, maturity model is a SPI solution or model that describes a set of levels or steps to allow maturing in a given software process;

- Adoption: Related to ASD adoption itself, tailoring, customization, adaptation, transition, etc.;

- Agile Improvement Solution (AIS): describes a solution (i.e., model, process, approach, framework, method, etc.) that is focused (i) on the definition of agile maturity levels or a maturity model itself; (ii) on the maturity assessment of a given company, team, etc.; (iii) on the adoption of ASD by a company, team, etc. For a solution to be considered an AIS, it must not be combined with traditional approaches. 
Following the definition of ASD maturity stated by Fontana et al. [11], researchers have proposed maturity models and agile maturity assessment solutions. According to Buglione [13], to be suitable for agile environments, agile maturity models should be inexpensive, fast and easy to understand, should produce short management reports, and should provide relevant drivers and best practices for a road map to maturity.

The goal of this study is to report the state of the art of AIS. We focus on the following questions: (i) do they consider agile teamwork factors?; (ii) were they applied to industry projects?; (iii) were they evaluated in terms of benefits of adoption such as increase on productivity, value delivered or cost reduction?; and (iv), do they provide tool support?

For this purpose, we performed a systematic literature reviewed following the guidelines proposed by Kitchenham and Charters [14] and Wohlin [15]. In this paper, we detail our study and also point the gaps and future directions for research in AIS.

The remaining of the paper is structured as follows: in Section II, we discuss previous literature reviews that are related to this study; in Section III, we describe the protocol of our review process; in Section IV, we present our findings; in Section V, we discuss the results; the threats to validity are presented in Section VI; and in Section VII, we present our conclusions and future works

\section{RELATED REVIEWS}

Some literature reviews were conducted on topics related to this review. We summarize them as follows.

Henriques \& Tanner [16], performed a systematic review of agile and maturity model research. The authors identified 39 relevant papers to their research, which aimed to identify the trends in research concerned with agile methods in the context of agile maturity models. They concluded that agile and CMMI can coexist when agile is introduced into already highly mature environments or when the primary goal is focused solely on the delivery. However, they concluded that if higher levels of CMMI maturity is the goal, agile cannot be used without being supplemented with other non-agile practices.

Silva et al. [17] evaluated, synthesized, and presented results on the use of ASD with CMMI. From 81 included studies, they concluded that agile methodologies can be used by companies to reduce efforts in getting to levels 2 and 3 of CMMI, there even being reports of applying agile practices that led to achieving level 5. However, as Henriques \& Tanner [16], they concluded that agile practices alone could not achieve level 5, being necessary to resort to additional practices.

Martinez et al. [18] identified the problems related to agile adoption in a systematic review, and stated that the findings of this review would be the basis to propose a framework to support the agile adoption. 27 papers were selected on their research after being filtered by the selection criteria. The authors categorized the problems in four groups: people, process, project, and company. Considering their findings, the literature reports more problems related to people.
Dikert et al. [19] conducted a systematic literature review of industrial large-scale agile transformations, and analyzed 52 papers describing 42 organizations. Part of the results of this review indicated that large-scale agile seems to be harder to implement than people expect, as companies complain about not finding enough guidance in the literature. The most challenge topics are integrating non-development functions, resistance to change, and requirements engineering.

Unlike the previously mentioned works, which cover the coexistence of agile and CMMI and problems related to agile adoption, we focus on the fidelity of AIS to the Agile Manifesto [20] in regards to teamwork factors (six out of the twelve principles are related to the individuals involved in the product development), their validity in real projects, as well as if they provide tool support to facilitate their usage by practitioners.

\section{Methodology}

In this research, we performed a Systematic Literature Review (SLR) following the guidelines presented in [14].

\section{A. Research Questions}

As previously mentioned, the main goal of this research is to evaluate the fidelity of AIS to the Agile Manifesto [20] in regards to teamwork factors, their validity in real projects, as well as if they provide tool support to facilitate their usage by practitioners. Therefore, we formulated the following research questions (RQ):

RQ1: What is the percentage of AIS that take teamwork factors in consideration?

RQ2: What is the percentage of AIS that were validated in real projects?

RQ3: Within the AIS validated in real projects, what is the percentage of them that present traces of increased efficiency/performance/productivity, or even cost reduction?

RQ4: What is the percentage of AIS that provide tool support?

\section{B. Identifying Primary Studies}

In order to identify the relevant primary studies for our research questions, we decided to use the snowballing guidelines defined in [15]. The start set of primary studies necessary for the snowballing was defined based on relevant studies identified in [16], and previously known studies by the authors. With these two sources, we had 53 studies - 39 from [16] and 14 previously know - to analyze according to the selection criteria we defined. From these 53 studies, we identified 22 relevant studies that composed the start set of the snowballing.

After having the start set defined, we started performing the snowballing iterations by executing the backward and forward snowballing steps, and applying the inclusion and exclusion criteria, as well as checking for duplicates until the moment that no relevant studies were found.

A paper is considered irrelevant in case it is (i) not related to ASD only, which means that we are not interested 
in studies that propose the usage of agile and traditional software development approaches (e.g., coexistence of agile and CMMI); (ii) not written in english; (iii) published in non peer reviewed publication channel such as books, thesis or dissertations, tutorials, keynotes, etc.; (iv) secondary study; and (v) duplicated. On the other hand, a relevant paper must present a solution that fits the description of AIS and is focused on ASD only.

Every paper identified in this review was evaluated based on the sequence of the four steps described below:

1) Initial Evaluation: Each paper found in the backward and forward snowballing steps were initially evaluated based on their titles, abstracts, and keywords. The goal of this step is to exclude every paper that does not fit this review context, and to not discard possible relevant papers;

2) Check of Duplicates: The goal of this step is to avoid rework. If a given paper is possibly relevant according to the Initial Evaluation, the reviewer needs to check in the database if that paper was already evaluated. The paper only goes to the next step of evaluation if it was not already in the database;

3) Superficial Evaluation: In this step, the reviewer needs to superficially check the paper in order to identify a solution that fits the review context. In case the paper presents no solution, the paper should be discarded. However, if it is not clear to the reviewer that the paper is relevant, it should not be discarded. Each paper is evaluated by two random reviewers in this step by following the procedure presented by Ali et al. [21];

4) Data Extraction: The goal of this step is to minutely evaluate the paper and extract the relevant information to answer the research questions. It is also possible to find irrelevant papers in this step because a deeper analysis is made if compared to the one made in the previous step. Two random reviewers are necessary to execute this step. The first reviewer is called data extractor and the second reviewer is called data checker. The role of the data extractor is to extract the relevant data from the paper if it is considered relevant. The data checker needs to check if every piece of data that was extracted from the paper is correct, and even if the paper is relevant or not depending on the data extractor's judgment. In case they do not reach a common sense, a third reviewer is invited to help them.

According to Ali et al. [21], there are six categories of agreement or disagreement between the reviewers, as shown in Figure 1. These categories were introduced on the third and fourth steps.

Categories A or B mean that at least one reviewer evaluated the paper as relevant and it is included. Category B occurs when one reviewer is uncertain about the relevance of the paper. To minimize the risk of discarding a significant study, the paper is included in the pool for the next step. Afterwards, in the next step all doubts about the paper's relevance are

\begin{tabular}{|c|c|c|c|}
\hline & \multicolumn{3}{|c|}{ Reviewer 2} \\
\hline & Relevant & Uncertain & Irrelevant \\
\hline Relevant & A & B & D \\
\hline Uncertain & B & $\mathrm{C}$ & E \\
\hline Irrelevant & D & E & F \\
\hline
\end{tabular}

Fig. 1. Categories of agreement or disagreement.

clarified with a further evaluation. Category $\mathrm{C}$ means that no concrete decision was made by any of the two reviewers and further investigation is needed. In this case, a third reviewer needs to evaluate the paper. If the third reviewer evaluates the paper as irrelevant, it is discarded; otherwise, it is included in the pool for the next step

Categories D and E are results from disagreement and the reviewers are asked to discuss what reasons led them to their respective decisions. After that, a consensus is expected and a new category (A, C or F) classification must be done. Papers in category $\mathrm{F}$ are excluded, as both reviewers agreed on their irrelevance.

\section{Extracted Data}

We used a spreadsheet editor to record information. For each paper, we extracted general information such as year of publication, and type of article, as well as data related to the RQ. The following data were extracted from the papers:

(i) year of publication;

(ii) type of article (i.e., journal, conference, or workshop);

(iii) validation context (i.e., none, academic, industrial, or both academic and industrial);

(iv) agile method domain;

(v) tool support;

(vi) considers teamwork factors

(vii) category (i.e., assessment, maturity model, adoption);

(viii) traces of increased efficiency/performance.

Some AIS are approached in more than one paper. For example: an AIS was introduced in a paper, and its validation is described in another paper. For this reason, the extraction of the data regarding the RQ, which is related to the AIS themselves and not just papers, was made by taking in consideration the set of papers that is related to a given AIS. Moreover, if an author uses an existing AIS as basis for another AIS by performing small modifications, it is considered a new AIS.

\section{RESULTS}

In this section, we present the results for the SLR process and for the RQ.

From the 22 papers that composed the start set, we identified 16 relevant papers by executing the snowballing steps. We used these 16 papers as the seed set for a new snowballing iteration and identified 11 papers. For the next iteration, we found 5 papers. During the last iteration, we found an additional 


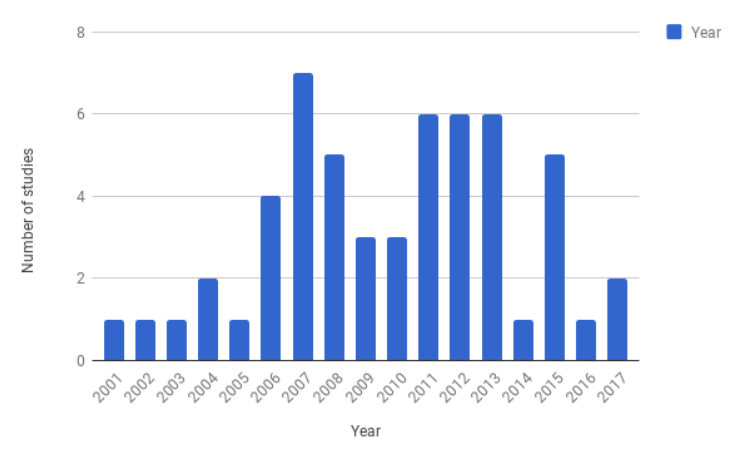

Fig. 2. Number of papers per year.

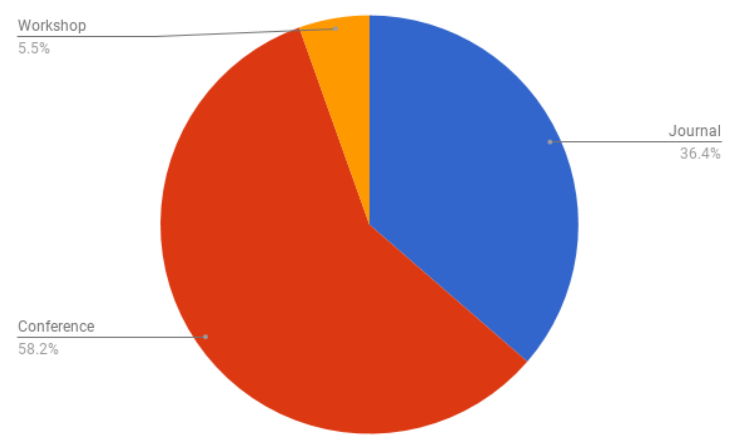

Fig. 3. Percentage of papers per type of publication channel.

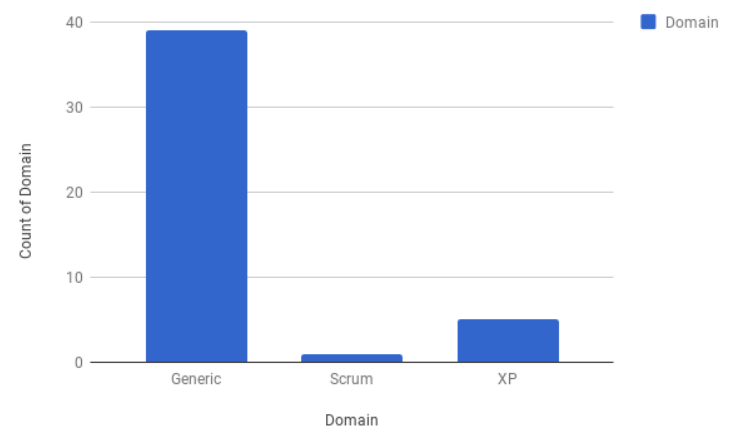

Fig. 4. Percentage of agile methods approached in the AIS.

paper. In total, we selected 55 papers. Due to space limitations, we present data extracted from 18 papers in Table I related to the RQ. The complete list of papers with more detailed information is available at https://goo.gl/FYoZYg.

In Figure 2 we present the amount of papers per year. In Figure 3 we show the percentage of papers per type of publication channel.

We identified 45 AIS by analyzing the papers and authors. In Figure 4, we present the distribution of the agile methods domain approached by the AIS. In Figure 5, we present the distribution of the AIS categories.

The percentage of AIS that consider teamwork factors in their approach is $60 \%$, which corresponds to 27 of the 45

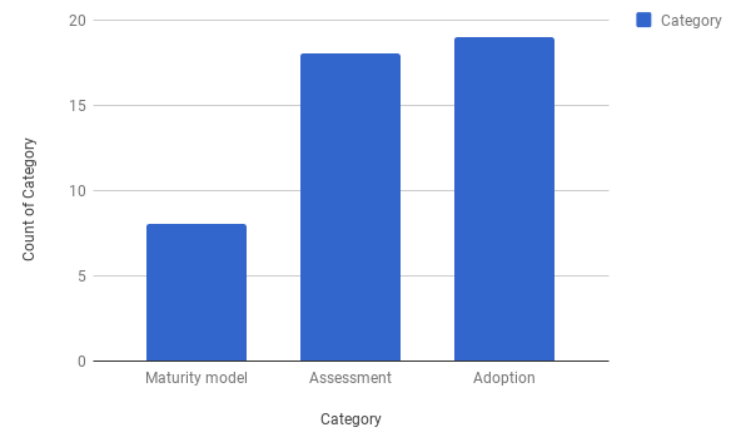

Fig. 5. Distribution of the AIS categories.

identified AIS. 21 solutions were validated in industry environments, which corresponds to $46.7 \%$ of the total. From these 21 AIS validated in industrial environments, only 10 presented benefits of their adoption such as increase on productivity, value delivered or cost reduction, which corresponds to $45.5 \%$. Finally, in regards to RQ4, only $28.9 \%$ of the identified AIS provide tool support, which corresponds to 13 AIS. These percentages, which are related to the $\mathrm{RQ}$, are also presented in Table II.

\section{DISCUSSION}

In this section, we discuss the results regarding the four research questions (see Section III-A).

The first paper identified was published in 2001, but we also identified two papers published in 2017. The period with the highest rate of publications was between 2006 and 2013. However, over the last five years, there was a significant number of papers published. We believe that these new solutions are being proposed because there is no consolidated basis to provide a strong theoretical foundation for the AIS, or most researchers do not want to build their solutions on top of such basis.

In regards to RQ1, $40 \%$ of the solutions identified in this research do not take teamwork factors in consideration. We consider this percentage very high given that the focus of this research is AIS. It is contradictory the fact that some solutions state they are focused on ASD, but do not approach important and valuable principles stated in the Agile Manifesto [20]. The lack of AIS that take teamwork factors in consideration is often identified in solutions that focuses on agile practices and objectives.

RQ2 is answered according to validation of the AIS on industry environments. The rate of solutions that were not validated in real projects is higher than $50 \%$. This percentage shows that there is a big gap between what the AIS identified promise and their application in real environments. Moreover, as discussed before, it is possible to argue that the lack of validation of these solutions may be a crucial factor contributing to the absence of a consolidated basis. The reason to believe in such a statement is very clear: if there is no data regarding the approval of these solutions in the industry, how would these 
TABLE I

EXTRACTED DATA RELATED TO RQ FROM 18 PAPERS.

\begin{tabular}{|c|c|c|c|c|}
\hline Title & Teamwork Factors & Validated in Industry & Evidence of Benefits & Tool Support \\
\hline $\begin{array}{l}\text { Progressive outcomes: A framework for maturing in } \\
\text { agile software }\end{array}$ & 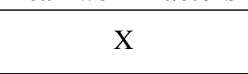 & 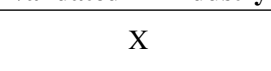 & - & \\
\hline \multicolumn{5}{|l|}{ Scrum Maturity Model } \\
\hline $\begin{array}{l}\text { Agile maturity model (AMM): A software process } \\
\text { improvement framework for agile software development } \\
\text { practices }\end{array}$ & $\mathrm{X}$ & & & $\mathrm{X}$ \\
\hline $\begin{array}{l}\text { Agile Compass: A Tool for Identifying Maturity in Agile } \\
\text { Software Development Teams }\end{array}$ & $\mathrm{X}$ & & & $\mathrm{X}$ \\
\hline \multicolumn{5}{|l|}{ Light maturity models (LMM): an Agile application } \\
\hline $\begin{array}{l}\text { An evaluation of the degree of agility in six agile methods } \\
\text { and its applicability for method engineering }\end{array}$ & $\mathrm{X}$ & & & $\mathrm{X}$ \\
\hline Rebalancing Your Organization's Agility and Discipline & $\mathrm{X}$ & & & $\mathrm{X}$ \\
\hline A framework for adapting agile development methodologies & $\mathrm{X}$ & & & \\
\hline Adopting agile in distributed development & & $\mathrm{X}$ & $\mathrm{X}$ & $\mathrm{X}$ \\
\hline $\begin{array}{l}\text { Project agility assessment: An integrated decision analysis } \\
\text { approach }\end{array}$ & & $\mathrm{X}$ & $\mathrm{X}$ & \\
\hline $\begin{array}{l}\text { An Approach for Assessing Suitability of Agile Solutions: } \\
\text { A Case Study }\end{array}$ & & $\mathrm{X}$ & & \\
\hline $\begin{array}{l}\text { A disciplined approach to adopting agile practices: The } \\
\text { agile adoption framework }\end{array}$ & & & & $\mathrm{X}$ \\
\hline $\begin{array}{l}\text { An iterative improvement process for agile software } \\
\text { development }\end{array}$ & & & $\mathrm{X}$ & \\
\hline $\begin{array}{l}\text { Adapting the Lean Enterprise Self-Assessment Tool for } \\
\text { the Software Development Domain }\end{array}$ & $\mathrm{X}$ & $\mathrm{X}$ & & $\mathrm{X}$ \\
\hline $\begin{array}{l}\text { Tailoring for agile methodologies: a framework for } \\
\text { sustaining quality and productivity }\end{array}$ & $\mathrm{X}$ & $\mathrm{X}$ & & \\
\hline Agile Transition Model Based on Human Factors & $\mathrm{X}$ & & & \\
\hline $\begin{array}{l}\text { A Mapping Model for Transforming Traditional Software } \\
\text { Development Methords to Agile Methodology }\end{array}$ & $\mathrm{X}$ & & & \\
\hline $\begin{array}{l}\text { AM-QuICk: A Measurement-Based Framework for Agile } \\
\text { Methods Customisation }\end{array}$ & $\mathrm{X}$ & $\mathrm{X}$ & & \\
\hline
\end{tabular}

TABLE II

RQ RELATED RESULTS

\begin{tabular}{|ccc|}
\hline Research Question & Percentage & Quantity \\
\hline Teamwork Factors & $60 \%$ & 27 \\
Validated in Industry & $46.7 \%$ & 21 \\
Evidence of Benefits & $45.5 \%$ & 10 \\
Tool Support & $28.9 \%$ & 13 \\
\hline
\end{tabular}

solutions evolve and serve as basis for future researches that pursue to solve similar problems?

$48.9 \%$ of the solutions that were validated, but $2.2 \%$, which correspond to 1 within the 45 identified AIS, were validated in Academic context. The percentage of validated AIS correspond to 21 within the 45 identified AIS. From these 21 AIS, $45.5 \%$ percentage of them do not present traces of increased efficiency/performance/productivity or cost reduction. In other words, almost half of them do not prove that there is a gain when applying such a solution in an industry environment. This percentage is an important indicator to explicit the lack of contributions these solutions provide to the industry environment. Some researchers may argue that they received positive feedback from the subjects involved on the validation of their AIS. However, these feedback could not provide substantial conclusion as a numeric evidence (e.g., based on the comparison between the effort to implement and use an AIS in a specific context, and the gains it provides). These discussion regarding $\mathbf{R Q 3}$ explicit the need for a better collaboration and proximity between the academic and industrial environments. This proximity could be crucial to obtain a more consolidated concept of agile maturity.

The percentage of AIS related to RQ4 indicates that only $23.8 \%$ of the included AIS provide tool support. This small percentage is another indicator of the existing gap between the industry and the academic community. Nowadays, there is a problem when trying to implement solutions proposed by the academic community into the industry. We believe it occurs because, usually, researches do not try to address their solutions to a more practical utility instead of solving a small problem with an approach that requires too much effort. Industry practitioners want tools, solutions, and instruments, that can make their work easier without requiring them to put more effort than they actually do.

\section{THREATS TO VALIDITY}

As well as in all SLR studies, a common threat to validity regards to the covering of all relevant studies. Therefore, to mitigate this problem, we executed the snowballing technique, as described in [15], until no more relevant papers were found.

Another threat is related to the researchers' opinions and judgments in regards to the extraction of the data, which can influence the results of the study. To mitigate this problem, each paper was evaluated by two reviewer. Also, depending on the disagreement between two reviewers, a third random 
reviewer was invited to the process, as explained in Section III-B.

\section{CONCLUSION}

In this paper, we conducted a SLR to investigate if AIS take in consideration important agile teamwork key factors; if they were applied in real projects; if, after applying them, it was possible to identify increases on efficiency/performance/productivity, or even cost reduction (i.e., something that proves that applying a given AIS brings real earnings besides positive feedback of subjects); and whether they provide tool support for applying them in real projects or not.

To reach this goal, we executed the snowballing procedure [15] on a start set containing 22 papers. From these 22 papers, we were able to identify other 33 relevant papers, resulting in a total of 55 included papers. From these 55 relevant papers, we identified 45 different AIS.

The results of this SLR indicate some of the possible reasons behind the lack of usage of academic proposed solutions in the industry environment. Most of the AIS investigated in this research were not validated in real projects, and do not provide tool support to facilitate their adoption. Moreover, almost half of the investigated that were validated do not present any trace of increased efficiency/performance/productivity, or even cost reduction.

Another issue identified in regards to the investigated AIS is that a high percentage of them do not present fidelity to the Agile Manifesto [20] because they do not take in consideration agile teamwork factors.

For future works, we intend to perform a more detailed analysis of the papers to understand the structure (e.g., levels, steps, stages, components, etc.) of the AIS, and perform a detailed comparison between them by category (i.e., maturity model, assessment, adoption). We also intend to complement the database by executing the forward snowballing on the relevant papers identified so far.

\section{ACKNOWLEDGMENT}

The authors would like to thank CAPES for supporting this research.

\section{REFERENCES}

[1] W. Alsaqaf, M. Daneva, and R. Wieringa, "Quality requirements in largescale distributed agile projects - a systematic literature review," in $R e$ quirements Engineering: Foundation for Software Quality, P. Grünbacher and A. Perini, Eds. Cham: Springer International Publishing, 2017, pp. 219-234.

[2] O. Salo and P. Abrahamsson, "An iterative improvement process for agile software development," Software Process: Improvement and Practice, vol. 12, no. 1, pp. 81-100, 2007. [Online]. Available: http://dx.doi.org/10.1002/spip.305

[3] I. Aaen, J. Arent, L. Mathiassen, and O. Ngwenyama, "A conceptual map of software process improvement," Scandinavian Journal of Information Systems, vol. 13, pp. 123-146, Jun. 2001. [Online]. Available: http://dl.acm.org/citation.cfm?id=565431.565437

[4] K. Lukasiewicz and J. Miler, "Improving agility and discipline of software development with the scrum and cmmi," IET Software, vol. 6, no. 5, pp. 416-422, October 2012.
[5] S. W. Baker, "Formalizing agility, part 2: how an agile organization embraced the cmmi," in AGILE 2006 (AGILE'06), July 2006, pp. 8 pp. -154 .

[6] C. R. Jakobsen and K. A. Johnson, "Mature agile with a twist of cmmi," in Proceedings of the Agile 2008, ser. AGILE '08. Washington, DC, USA: IEEE Computer Society, 2008, pp. 212-217. [Online]. Available: https://doi.org/10.1109/Agile.2008.10

[7] J. Sutherland, C. R. Jakobsen, and K. Johnson, "Scrum and cmmi level 5: The magic potion for code warriors," in Agile 2007 (AGILE 2007), Aug 2007, pp. 272-278.

[8] S. Cohan and H. Glazer, "An agile development team's quest for $\mathrm{cmmi}$ maturity level 5," in 2009 Agile Conference, Aug 2009, pp. 201-206.

[9] D. J. Anderson, "Stretching agile to fit cmmi level 3 - the story of creating msf for cmmi reg; process improvement at microsoft corporation," in Agile Development Conference (ADC'05), July 2005, pp. 193-201.

[10] M. C. Paulk, "Extreme programming from a $\mathrm{cmm}$ perspective," IEEE Software, vol. 18, no. 6, pp. 19-26, Nov 2001.

[11] R. M. Fontana, I. M. Fontana, P. A. da Rosa Garbuio, S. Reinehr, and A. Malucelli, "Processes versus people: How should agile software development maturity be defined?" Journal of Systems and Software, vol. 97, pp. 140 - 155, 2014.

[12] M. Kohlegger, R. Maier, and S. Thalmann, "Understanding maturity models results of a structured content analysis," in Proceedings of IKNOW '09 and I-SEMANTICS '09, September 2009, pp. 193-201.

[13] L. Buglione, "Light maturity models (lmm): An agile application," in Proceedings of the 12th International Conference on Product Focused Software Development and Process Improvement, ser. Profes '11. New York, NY, USA: ACM, 2011, pp. 57-61. [Online]. Available: http://doi.acm.org/10.1145/2181101.2181115

[14] B. Kitchenham and S. Charters, "Guidelines for performing Systematic Literature Reviews in Software Engineering," Keele University and Durham University Joint Report, Tech. Rep. EBSE 2007-001, 2007.

[15] C. Wohlin, "Guidelines for snowballing in systematic literature studies and a replication in software engineering," in Proceedings of the 18th International Conference on Evaluation and Assessment in Software Engineering, ser. EASE '14. New York, NY, USA: ACM, 2014, pp. 38:1-38:10. [Online]. Available: http://doi.acm.org/10.1145/2601248. 2601268

[16] M. T. Vaughan Henriques, "A systematic literature review of agile and maturity model research," Interdisciplinary Journal of Information, Knowledge, and Management, vol. 12, pp. 53-73, 2017. [Online]. Available: http://www.ijikm.org/Volume12/ IJIKMv12p053-073Henriques3025.pdf

[17] F. S. Silva, F. S. F. Soares, A. L. Peres, I. M. de Azevedo, A. P. L. Vasconcelos, F. K. Kamei, and S. R. de Lemos Meira, "Using cmmi together with agile software development: A systematic review," Information and Software Technology, vol. 58, pp. $20-43$, 2015. [Online]. Available: http://www.sciencedirect.com/science/article/ pii/S0950584914002110

[18] J. López-Martínez, R. Juárez-Ramírez, C. Huertas, S. Jiménez, and C. Guerra-García, "Problems in the adoption of agile-scrum methodologies: A systematic literature review," in 2016 4th International Conference in Software Engineering Research and Innovation (CONISOFT), April 2016, pp. 141-148.

[19] K. Dikert, M. Paasivaara, and C. Lassenius, "Challenges and success factors for large-scale agile transformations: A systematic literature review," Journal of Systems and Software, vol. 119, pp. 87 - 108, 2016. [Online]. Available: http://www.sciencedirect.com/science/article/ pii/S0164121216300826

[20] K. Beck, M. Beedle, A. van Bennekum, A. Cockburn, W. Cunningham, M. Fowler, J. Grenning, J. Highsmith, A. Hunt, R. Jeffries, J. Kern, B. Marick, R. C. Martin, S. Mellor, K. Schwaber, J. Sutherland, and D. Thomas, "Manifesto for agile software development," http://www. agilemanifesto.org/, 2001.

[21] N. B. Ali, K. Petersen, and C. Wohlin, "A systematic literature review on the industrial use of software process simulation," Journal of Systems and Software, vol. 97, pp. 65 - 85, 2014. [Online]. Available: http://www.sciencedirect.com/science/article/pii/S0164121214001502 